\title{
Expression Pattern and Prognostic Significance of Claudin 1, 4 and 7 in Pancreatic Cancer
}

\author{
Arsenal Sezgin Alikanoglu1*, Seyda Gunduz², Ozlem Demirpence ${ }^{3}$, Dinc Suren', \\ Umut Riza Gunduz ${ }^{4}$, Cem Sezer ${ }^{1}$, Mustafa Yildiz ${ }^{2}$, Mustafa Yildirim ${ }^{5}$
}

\begin{abstract}
Background: Tight junctions (TJs) organise paracellular permeability and they have an important role in epithelial and endothelial cell polarity and permanence of barrier function. It has been demonstrated that the Claudin family constitutes an important component of them. In this study, we assessed expression patterns of of Claudin1, 4 and 7 and whether they have any relation with prognosis in patients with pancreatic cancer. Materials and Methods: Expression patterns of Claudin 1,4 and 7 were examined by immunohistochemistry in 25 patients with a histopathological diagnosis of pancreatic cancer using a semiquantitative scoring of the extent and intensity of staining. After grouping the staining scores as low (final score 0-2) and high (final score 3-9) the relation between expression of Claudin 1,4 and 7 and survival was evaluated. Results: There was no significant relation between expression of Claudin 1,4 and 7 and gender and stage. No statistically significant relation was found between Claudin 1 and 4 expression and survival whereas a statistically significant relation was found between decrease in Claudin 7 expression and decrease in survival. Conclusions: Claudins have important functions other than their popular function known as adhesion. Supporting this hypothesis, we found a statistically significant relationship between increased Claudin 7 expression and increased survival time, and this suggests that Claudin 7 may exert different tumorigenic effects in pancreatic cancer other than its wellknown adhesion effect.
\end{abstract}

Keywords: Claudin 1 - Claudin 4 - Claudin 7 - pancreatic cancer - survival - tight junctions

Asian Pac J Cancer Prev, 16 (10), 4387-4392

\section{Introduction}

Tight junctions (TJs) organise paracellular permeability and they have an important role in epithelial and endothelial cell polarity and permanence of barrier function. As a result of many studies on the molecular ecture of TJs, it has been demonstrated that the Claudin family constitutes an important component of them. There are 24 known members of the Claudin family, each with a specific distribution pattern (Rahner et al., 2001; Tsukita et al., 2001).

Claudin 1,4,5,7,8,11,14 and 19 are impermeability Claudins and increase in expression of these Claudins is known to strengthen the junction of epithelial cells (Oliveira et al., 2007; Krause et al., 2008; Will et al., 2008; Ouban et al., 2010). Increased expression of the pore-forming Claudins 2,10 and 16 reduces the junction of epithelial cells (Bornholdt et al., 2011). Other Claudins have the ability of forming paracellular anion/cation pores and water channels (Tsukita et al., 2001; Will et al., 2008).

Loss of cell-to-cell adhesion is known as an important factor in cellular transformation and tumours' gaining metastatic potential (Fritzsche et al., 2008). It has been recently demonstrated that the Claudin protein family plays an important role in a series of pathophysiological events including development of cancer (Singh et al., 2010).

Claudin 1,4 and 7 are important members of this Claudin protein family and their expressions show alterations in many different malignancies such as oesophageal, gastric, biliary (Usami et al., 2006; Agarwal et al., 2009; Kwon et al., 2011), ovarian (Kwon et al., 2010), endometrial (Pan et al., 2007; Knoecny et al., 2008), bladder (Boireau et al., 2007; Szekely et al., 2011) renal (Lechpammer et al., 2008), prostate (Landers et al., 2008), breast (Kominsky et al., 2003), cholangiocarcinoma (Bunthot et al., 2012) and pancreatic cancer.

Pancreatic cancer is one of the most aggressive cancers with a strong capacity of invasion, metastasis and recurrence and known to be the fourth leading cause of cancer-related death in the industrialised world (Eskelinen et al.,1999; Yeo et al., 2002; Jemal et al., 2010). Despite multimodal therapeutic approaches including surgery, chemotherapy, radiotherapy, and immunotherapy, pancreatic cancer still presents a fatal prognosis (Tsutsumi et al., 2012). Development of new strategies for diagnosis

${ }^{1}$ Department of Pathology, ${ }^{2}$ Department of Medical Oncology, ${ }^{4}$ Department of General Surgery, Antalya Education and Research Hospital, Antalya, ${ }^{3}$ Department of Biochemistry, Cumhuriyet University Medical Faculty, Sivas, ${ }^{5}$ Department of Medical Oncology, Gaziantep Medical Park Hospital, Gaziantep, Turkey*For correspondence: arsi75@hotmail.com 
and therapy is neccessary to obtain a decrease in mortality (Kojima et al., 2012; Neesse et al., 2013; Wang et al., 2014). Recently, it was found that in several human cancers, including pancreatic cancer, some members of the claudin family are regulated abnormally and therefore are promising molecular targets for diagnosis and therapy (Michl et al., 2003; Kojima et al., 2012).

In this study, we searched the expression patterns of of Claudin1,4 and 7 and if they have any relation with prognosis in patients with pancreatic cancer.

\section{Materials and Methods}

\section{Patient selection}

Patients who were histopathologically diagnosed pancreatic ductal adenocarcinoma and in follow-up in Antalya Education and Research Hospital between January 2013 and October 2013 were enrolled in this study. Patients who were staged according to radiologic and clinical findings were re-staged according to the 7th edition of the American Joint Committee on Cancer. Finally a total of 25 patients with appropriate tissue samples were included in the study. Claudin 1, 4 and 7 expressions were examined by immunohistochemical method in biopsy specimens of these 25 patients. Demographic data such as age, gender and stage of the disease and information about the treatment applied were obtained by searching the patient files.

\section{Tissue preparation and immunohistochemical staining}

Biopsy materials obtained for histopathological diagnosis were placed in $10 \%$ formaldehyde immediately after the process and fixed for $24 \mathrm{~h}$. After fixation, materials were grossly examined and appropriately sampled. Tissue samples were embedded in paraffin after routine tissue procedure. Immunohistochemical staining was applied on cross-sections obtained from the tissue samples with tumour chosen after evaluating the hematoxylin and eosin stained slides. Cross-sections of $4-\mu \mathrm{m}$ thickness prepared for immunohistochemical staining were deparaffinised in an oven at $60^{\circ} \mathrm{C}$ for $2 \mathrm{~h}$. Subsequently, they were kept in xylene for $30 \mathrm{~min}$ and $100 \%$ alcohol for 30 min, and then washed with water. Slides were kept in a solution buffered with $10 \%$ citrate in the microwave at maximum power ( 800 watts) for $15 \mathrm{~min}$. Afterwards, the power was decreased by half for an additional $20 \mathrm{~min}$ in the microwave. Slides brought out of the microwave were kept at room temperature for $20 \mathrm{~min}$. Endogenous peroxidase activity was removed by being kept in $3 \%$ hydrogen peroxide for $10 \mathrm{~min}$. After being kept in primary antibodies Claudin 1 (rabbit polyclonal, clone ab15098, dilution 1:200, Abcam, Cambridge, MA, USA), Claudin 4 (rabbit polyclonal, clone ab15104, dilution 1:200, Abcam, Cambridge, MA, USA), and Claudin 7 (rabbit polyclonal, clone ab27487, dilution 1:200, Abcam, Cambridge, MA, USA) for $60 \mathrm{~min}$, they were washed in PBS for $5 \mathrm{~min}$. Afterwards, they were treated with biotinylated secondary antibody (Vector Laboratories, Burlingham, CA) for 20 min and washed with PBS for 5 min. They were kept with peroxidase conjugated antibody for $20 \mathrm{~min}$. Then they were washed in PBS for 5 min. They were kept in chromogenic DAB for 5 min. Slides were washed under tap water and then counterstained with hematoxylin. They were dehydrated, dried, and mounted with Entellan. For negative controls, the primary antibodies were omitted.

\section{Evaluation of immunohistochemically stained sections}

Expression rates of Claudin 1,4 and 7 in the tumor cells were evaluated by 2 pathologists (ASA, DS) who were unaware of the clinicopathological information of the patients. Only membranous staining was classified as positive, and in intensely stained cells, an accompanying cytoplasmic staining was also observed. Immunohistochemical evaluation and scoring was performed by the method that Hsueh et al. used (Hsueh et al., 2010). The expression of Claudins 1, 4, and 7 was assessed by semiquantitative scoring of the extent and intensity of the staining. The staining extent was represented by the percentage of positively stained tumor cells and graded as less than $10 \%(1+)$, in between $10 \%$ and $50 \%(2+)$, or more than $50 \%(3+)$, respectively. The staining intensity was recorded as absent $(0)$, weak $(1+)$, moderate $(2+)$, or strong $(3+)$, respectively. The 2 scores were multiplied to give a final score of 0 to 9 . The staining scores were grouped as low (final score 0-2) and high (final score 3-9). Figures 1,2 and 3 demonstrate examples of Claudin 1,4 and 7 staining representing different scores (Figure 1, 2, 3).

\section{Statistical analysis}

Statistical analyses were carried out by SPSS software for Windows 15.0. Suitability of variables to normal dispersion was observed by using visual (histograms and probability graphics) and analytical (Kolmogorov-Smirnov, and Shapiro- Wilk tests) methods. In Kolmogorov-Smirnov testing, $p$ values above 0.05 are considered as normal dispersion. Differences between groups were observed by using chi-square and MannWhitney U test. Kaplan-Meier survival analysis was performed for the relation of each immunohistochemical positive and negative result with survival. Statistical differences were confirmed by log-ranking test. $\mathrm{P}$ values under 0.05 were considered to be significant.

\section{Results}

\section{Clinicopathologic characteristics}

The study included $10(40 \%)$ female and $15(60 \%)$ male patients. The mean age of the patients was $68.9 \pm 8.8$ (range 51-89). Curative and palliative surgery was applied in $8(32 \%)$ and $3(12 \%)$ of the patients, respectively whereas diagnosis was made by histopathological evaluation of the biopsy material in $14(56 \%)$ of the patients.

The histologic type of the tumour was ductal adenocarcinoma in all of the patients. Differentiation degree of adenocarcinoma was identified as well, moderate and poor in $2(8 \%), 14(56 \%)$, and $9(36 \%)$ patients, respectively. When evaluated according to stage of the disease, $3(12 \%)$ patients were in stage 2, $7(28 \%)$ patients were in stage 3 and 15 (60\%) patients were in stage 4 .

\section{Immunohistochemical findings}


Claudin 1 expression was found as negative in 6 $(24 \%), 1(+)$ in $8(32 \%), 2(+)$ in $7(28 \%)$ and $3(+)$ in 4 $(16 \%)$ patients. Final score of Claudin 1 expression was found as low in $13(52 \%)$ and high in 12 (48\%) patients.

Claudin 4 expression was found as negative in $3(12 \%)$ $1(+)$ in $5(20 \%), 2(+)$ in $15(60 \%)$ and $3(+)$ in $2(8 \%)$ patients. Final score of Claudin 4 expression was found as low in $7(28 \%)$ and high in $18(72 \%)$ patients.

Claudin 7 expression was found as negative in $1(4 \%)$, $1(+)$ in $1(4 \%), 2(+)$ in $3(12 \%)$ and $3(+)$ in $20(80 \%)$ patients. Final score of Claudin 7 expression was found as low in $2(8 \%)$ and high in $23(92 \%)$ patients.

\section{Correlation between clinical parameters and expression} of claudins

There was no significant relation between Claudin 1 expression and gender and stage (p:0.87, p:0.063,). There was no relation between Claudin 4 expression and gender and stage (p:0.275, p:0.269). No relation was found between Claudin 7 expression and gender and stage (p:0.229, p:0.485).

The mean follow-up time was 9,8 months and

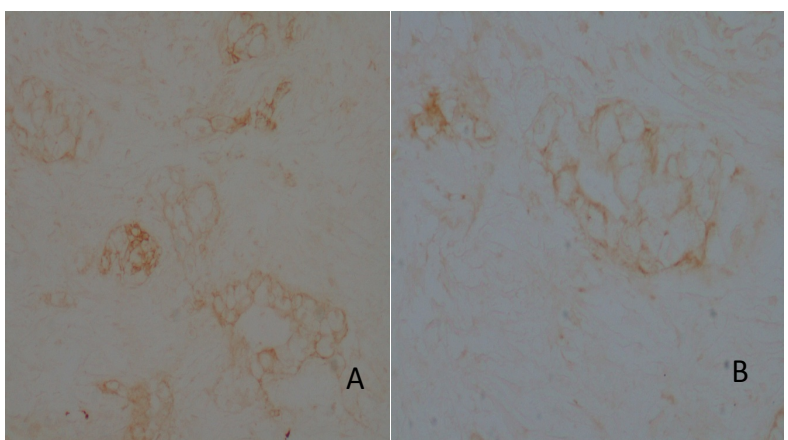

Figure 1A,1B. Weak Staining with Claudin 1 (IHC, $\mathrm{X} 200, \mathrm{X} 400$ )

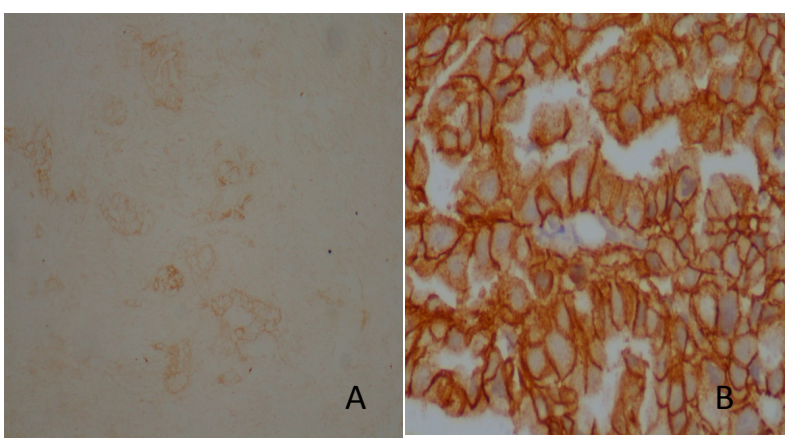

Figure 2A,2B. Moderate, Extensive and Strong Staining with Claudin 4 (IHC, Claudin 4 X100, X400)

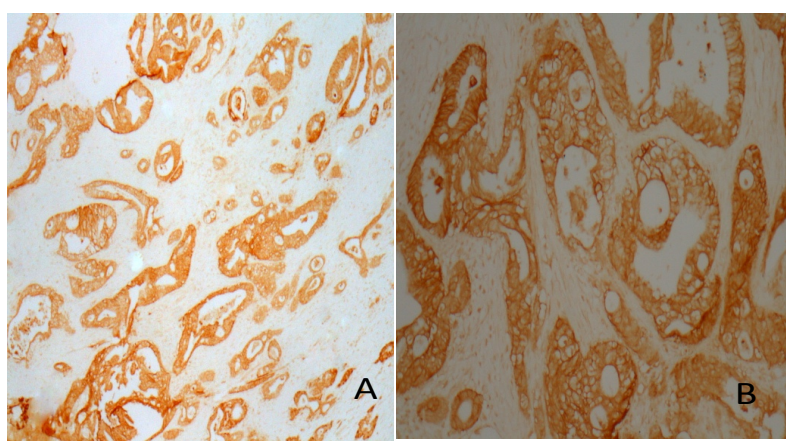

Figure 3A,3B: Extensive and Strong Staining with Claudin 7 (IHC, Claudin 7 X40, X100)

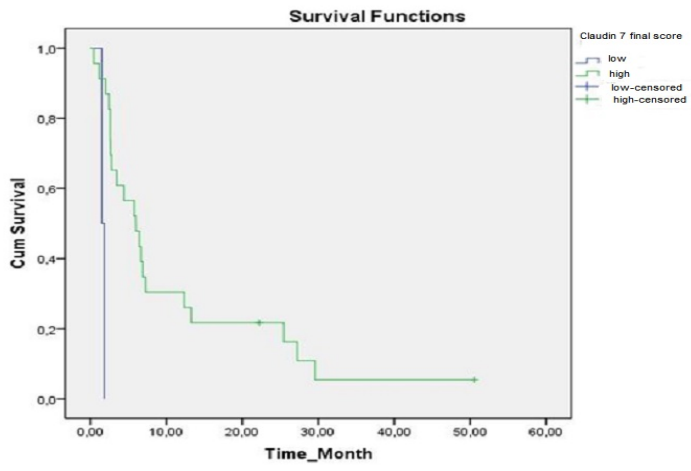

Figure 4. Relation between Decrease in Expression of Claudin 7 and Survival (p:0.001)

median follow-up time was 5.7 months. The 12-month and 24-month survival rates of the patients were found as $24 \%$ and $15 \%$, respectively. Median survival period was 5.7 \pm 2.1 months (\%95 Confidence İnterval 1.6-9.9). No statistically significant relation was found between Claudin 1 and 4 expression and survival (p:0.865, $\mathrm{p}: 0.947)$. Whereas a statistically significant relation was found between decrease in Claudin 7 expression and decrease in survival (p:0.001) (Figure 4).

\section{Discussion}

In our study, we showed that expression of Claudin 7 is a good prognostic factor in patients with pancreatic cancer whereas no association was found between prognosis and expression of Claudin 1 and 4 .

Loss of expression of Claudin is suggested to play a role in carcinogenesis by causing supression of TJs functions and subsequent cell proliferation, motility and invasiveness of the cancer cells (Rangel et al., 2003; Sheehan et al., 2007). On the other hand, although association between increase in Claudin expression and carcinogenesis is not clearly understood, it is suggested that matrix metalloproteinase activation due to overexpression of Claudin increases invasiveness of cancer (Singh et al., 2010). The role of Claudin expression in epithelial cell polarity, invasion and metastasis of cancer has been reported (Kojima et al., 2008; Yoon et al., 2010).

Subcellular distrubition and abundance of spesific claudin proteins are known to be different between normal and transformed pancreatic epithelia. Therefore it is possible that changes in paracellular permeability are associated with the formation of pancreatic intraepithelial neoplasms (PanIN) (Westmoreland et al., 2012).

In a study of Dehghan Esmatabadi, et al (2015), the effects of dendrosomal curcumin (DNC) on cellular migration and adhesion of human SW480 colon cancer cells were searched and DNC was found to inhibit metastasis by causing a decrease in mRNA levels of some proteins including Claudin 1, Hef 1 and Zeb 1 (Dehghan Esmatabadi et al., 2015).

Tan et al. (2004) showed that expression and dispersion of Claudin 1 is related with dissociation of the pancreatic cancer cells (Tan et al., 2004). In another study supporting this, Kyuno et.al reported that expression of Claudin 1 increases in epithelial-mesenchymal transition in 
pancreatic cancer and this increase can be reduced by protein kinase inhibitors (Kyuno et al., 2013). It is also shown that Claudin 1 expression induces cell growth by increasing expression of TNF- $\alpha$ in pancreatic cancer cell (Kondo et al., 2008).

Pancreatic ductal adenocarcinomas as well as intraductal papillary mucinous tumours of the pancreas express claudins 1 and 4, and claudin 18 is present mainly in pancreatic carcinoma (Tsukahara et al., 2005; Karanjawala et al., 2008).

Claudin-4 expression is widely dysregulated in epithelial malignancies and in a number of premalignant precursor lesions. It also seems to play an important role in tumour cell invasion and metastasis and its dual role as receptor of Clostridium perfringens enterotoxin (CPE) is very important in means of molecular targeted approaches. Upregulation of Claudin 4 in pancreatic cancer has been shown by using various methods for identifying expression profiles (Neesse et al., 2012).

Nichols et. al (2004) performed immunohistochemical evaluation of Claudin 4 in tissue samples of patients with primary and metastatic pancreatic cancer and foci of pancreatic intraepithelial neoplasia (PanIN) present in cases with pancreatic cancer in their study. Claudin 4 was found intensely positive in virtually all primary (71/72 [99\%]) and metastatic (49/49 [100\%]) pancreatic cancer tissue samples analyzed and in 10 of 11 samples of PanIN. These findings support the use of claudin 4 as a target for novel therapeutics of pancreatic cancer. Furthermore, the Claudin 4 overexpression within the precursor lesion of pancreatic cancer ; PanIN, suggests a potential benefit of demonstrating Claudin 4 expresion before the development of an invasive carcinoma (Nichols et al., 2004).

In a review of Kojima et al. (2012), studies concerning Claudin 4 in both normal epithelial cells of human pancreatic duct and cancer cells were discussed. Claudin-4, overexpressed in pancreatic cancer and its precursor lesions, is a receptor for Clostridium perfringens enterotoxin (CPE). It is suggested that PKC $\alpha$ inhibitors may represent potential therapeutic agents against human pancreatic cancer cells by the use of CPE cytotoxicity via claudin-4 (Kojima et al., 2012)

In our study 18 (72\%) of the patients presented a high final score of Claudin 4 expression. In a study of Tsutsumi, et al. (2012), Claudin 4 mRNA expression was searched by quantitative real-time reverse transcription- polymerase chain reaction (qRT-PCR) in a panel of 9 pancreatic cancer cell lines and immunohistochemical analysis. Increased expression of Claudin 4 was confirmed in all the pancreatic cancer cell lines tested compared with normal ductal epithelial cells and fibroblasts. A significant association was found between low expression of Claudin 4 and shorter survival in patients with pancreatic cancer. It was also reported that patients with high Claudin 4 expression survived longer than patients with low Claudin4. In immunohistochemical analysis, the level of Claudin 4 mRNA expression was significantly correlated with the expression of Claudin 4 protein (Tsutsumi et al., 2012).

In our study, no statistically significant relation was found between Claudin 4 expression and survival.
Although claudin-4 is considered to be highly expressed in many pancreatic carcinomas and several other solid tumors, Michl et. al reported in vitro and in vivo findings demonstrating claudin- 4 as an anti-invasive factor. On the basis of the immunohistochemical findings in their series of pancreatic carcinoma tissues, Claudin- 4 expression was found higher in well-differentiated carcinomas than in undifferentiated tumors. This effect was morphologically associated with increased formation of tight junctions between tumor cells. Claudin-4 is negatively regulated by TGF- $\alpha$ and down-regulated by inhibition of the Ras signaling pathway. In order to unequivocally prove a correlation among tumor grading, invasiveness, and claudin-4 expression, a study with a larger series of tumor specimens is warranted (Michl et al., 2003).

Claudins are found to be abnormally regulated in several human cancers including pancreatic cancer (Michl et al., 2003; Karanjawala et al., 2008).

On the other hand, in the development and progression of cancer, tumor suppressor genes may be silenced by mechanisms such as methylation. Silencing of the expression of some claudins in several human cancers is correlated with promoter hypermethylation. These include claudin-7 in breast, claudin-4 in bladder and claudin-11 in gastric cancer (Kominsky et al., 2003; Boireau et al., 2007; Agarwal et al., 2009).

In a study of a series of the rare pancreatic SPT (solid pseudopapillary tumour) Comper et al. (2009) showed that claudin expression profile of this tumor is interesting when compared with claudin expression of the normal pancreas, PET (pancreatic endocrine tumour), ACC (pancreatic acinar cell carcinoma (ACC) and and PB (pancreatoblastoma ) and that the pattern of claudins 5 and 7 expression is highly specific in differentiating SPT from PET, ACC, and PB (Comper et al., 2009).

In our study Claudin 7 expression was found as negative in $1(4 \%), 1(+)$ in $1(4 \%), 2(+)$ in $3(12 \%)$ and $3(+)$ in $20(80 \%)$ patients. Final score of Claudin 7 expression was found as low in $2(8 \%)$ and high in 23 $(92 \%)$ patients.

Soini, et al (2012) searched the expression of claudins 7 and 18 in pancreatic ductal adenocarcinoma in a total of 111 cases in their study and found that there was no association between expression of Claudin 7 and survival (Soini et al., 2012). In our study we found that decrease in expression of Claudin 7 has a statistically significant relation with decrease in survival (p:0.001). These discrepant results may be due to the difference between number and characteristics of the cases of the studies.

Borka et al. (2007) showed expression of Claudin 1,4 and 7 in pancreatic adenocarcinoma (Borka et al., 2007). Tsukahara et al. (2005) investigated the expression of claudin-4 in human pancreas, pancreatic ductal adenocarcinomas, and intraductal papillarymucinous tumors of the pancreas (IPMT), and compared with that of claudin-1. Of 12 cases of pancreatic ductal adenocarcinoma, $11(92 \%)$ had positive immunostaining for claudin- 4 , and seven (58\%) for claudin-1 (Tsukahara et al., 2005). We found a similiar expression rate for Claudin 1 whereas Claudin 4 expression rate was found higher in our study. This may be due to the higher number 
of patients in our study.

In conclusion, the main role of members of Claudin family is cell-to-cell adhesion. Therefore it is suggested that decrease in cellular expression of Claudins causes increased motility in the tumour cells and invasion. However, an increase in expression of Claudin family is observed in some types of cancer, surprisingly.

Various studies reported that some of the members of the Claudin family cause decrease in ability of invasion of tumour cells, in some types of cancer overexpression of Claudins cause a decrease in apoptosis and an increase in survival of the cell. And all of these findings indicate that Claudins have important functions other than their popular function known as adhesion. Supporting this hypothesis, we found a statistically significant relationship between increased Claudin 7 expression and increased survival time, and this suggests that Claudin 7 has different tumourogenic effects in pancreatic cancer other than its well-known adhesion effect.

Thus, we think that this specific member of Claudin family is a promising molecular marker for prognosis, diagnosis and therapy in pancreatic cancer.

\section{References}

Agarwal R, Mori Y, Cheng Y, et al (2009). Silencing of claudin-11 is associated with increased invasiveness of gastric cancer cells. PLoS One, $\mathbf{4}, 8002$.

Boireau S, Buchert M, Samuel MS, et al (2007). DNAmethylation-dependent alterationsof claudin- 4 expression in human bladder carcinoma. Carcinogenesis, 28, 246-58.

Borka K, Kaliszky P, Szabo E, et al (2007). Claudin expression in pancreatic endocrine tumors as compared with ductal adenocarcinomas. Virchows Arch, 450, 549-57.

Bornholdt J, Friis S, Godiksen S, et al (2011). The level of Claudin-7 is reduced as an early event in colorectal carcinogenesis. BMC Cancer, 11, 65.

Bunthot S, Obchoei S, Kraiklang R, et al (2012). Overexpression of claudin-4 in cholangiocarcinoma tissues and its possible role in tumor metastasis. Asian Pac J Cancer Prev, 13, 71-6.

Comper F,Antonello D, Beghelli S, et al (2009). Expression pattern of claudins 5 and 7 distinguishes solidpseudopapillaryfrom pancreatoblastoma, acinar cell and endocrinetumors of the pancreas. Am J Surg Pathol, 33, 768-74.

Dehghan Esmatabadi MJ, Farhangi B, Safari Z, et al (2015). Dendrosomal curcumin inhibits metastatic potential of human SW480 colon cancer cells through down-regulation of Claudin1, Zeb1 and Hef1-1 gene expression. Asian Pac J Cancer Prev, 16, 2473-81.

Eskelinen MJ, Haglund UH (1999). Prognosis of human pancreaticadenocarcinoma: review of clinical and histopathological variablesand possible uses of new molecular methods. Eur J Surg, 165, 292-306.

Fritzsche FR, Oelrich B, Johannsen M, et al (2008). Claudin-1 protein expression is a prognostic marker of patient survival in renal cell carcinomas. Clin Cancer Res, 14, 7035-42.

Hsueh C, Chang YS, Tseng NM, et al (2010). Expression pattern and prognostic significance of claudins 1,4 , and 7 innasopharyngeal carcinoma. Hum Pathol, 41, 944-50

Jemal A, Siegel R, Xu J, Ward E (2010). Cancer statistics. CA Cancer J Clin, 60, 277-300.

Karanjawala ZE, Illei PB, Ashfaq R, et al (2008). New markers of pancreatic cancer identified through differentialgene expression analyses: claudin 18 and annexin A8. Am JSurg Pathol, 32, 188-96.
Kojima T, Takano K, Yamamoto T, et al (2008). Transforming growth factor-beta induces epithelial to mesenchymal transition by down-regulation of claudin- 1 expression and the fence function in adult rat hepatocytes. Liver Int, $\mathbf{2 8}$, 534-45.

Kojima T, Sawada N (2012). Regulation of tight junctions in human normal pancreatic duct epithelial cells and cancer cells. Ann N Y Acad Sci, 1257, 85-92.

Kojima T, Kyuno D, Sawada N (2012). Targeting claudin-4 in human pancreatic cancer. Expert Opin Ther Targets, 16, 881-7.

Kominsky SL, Argani P, Korz D, et al (2003). Loss of the tight junction protein claudin-7 correlates with histological grade in both ductal carcinoma in situ and invasive ductal carcinoma of the breast. Oncogene, 22, 2021-33.

Kondo J, Sato F, Kusumi T, et al (2008). Claudin-1 expression is induced by tumor necrosis factor-alpha in human pancreatic cancer cells. Int J Mol Med, 22, 645-9.

Konecny GE, Agarwal R, Keeney GA, et al (2008). Claudin-3 and Claudin-4 expression in serous papillary, clear cell, and endometrioid endometrial cancer. Gynecol Oncol, 109, 263-9.

Krause G, Winkler L, Mueller SL, et al (2008). Structure and function of Claudins. Biochim Biophys Acta, 1778, 631-45.

Kwon MJ, Kim SS, Choi YL, et al (2010). Derepression of CLDN3 and CLDN4 during ovarian tumorigenesis is associated withloss of repressive histone modifications. Carcinogenesis, 31, 974-983.

Kwon MJ, Kim SH, Jeong HM, et al (2011). Claudin-4 overexpression is associated with epigenetic derepression in gastric carcinoma. Lab Invest, 91, 1652-67.

Kyuno D, Kojima T, Yamaguchi H, et al (2013). Protein kinase $\mathrm{C} \alpha$ inhibitor protects against downregulation of claudin-1 during epithelial-mesenchymal transition of pancreatic cancer. Carcinogenesis, 34, 1232-43.

Landers KA, Samaratunga H, Teng L, et al (2008). Identification of claudin-4 as a marker highly overexpressed in both primary and metastatic prostate cancer. Br J Cancer, 99, 491-501.

Lechpammer M, Resnick MB, Sabo E, et al (2008). The diagnostic and prognostic utility of claudin expression in renal cell neoplasms. Mod Pathol. 21, 1320-9.

Michl P, Barth C, Buchholz M, et al (2003). Claudin-4 expression decreases invasiveness and metastatic potential of pancreatic cancer. Cancer Res, 63, 6265-71.

Neesse A, Griesmann H, Gress TM, Michl P (2012). Claudin-4 as therapeutic target in cancer. Arch Biochem Biophys, 524, 64-70.

Neesse A, Hahnenkamp A, Griesmann H, et al (2013). Claudin4-targeted optical imaging detects pancreatic cancer and its precursor lesions. Gut, 62, 1034-43.

Nichols LS, Ashfaq R, Iacobuzio-Donahue CA (2004). Claudin 4 protein expression in primary and metastatic pancreatic cancer: support for use as a therapeutic target. Am J Clin Pathol, 121, 226-30.

Oliveira SS, Morgado-Diaz JA (2007). Claudins: multifunctional players in epithelial tight junctions and their role in cancer. Cell Mol Life Sci, 64, 17-28.

Ouban A, Ahmed AA (2010). Claudins in human cancer: a review. Histol Histopathol, 25, 83-90.

Pan XY, Wang B, Che YC, et al (2007). Expression of claudin-3 and claudin- 4 in normal, hyperplastic, and malignant endometrial tissue. Int J Gynecol Cancer, 17, 233-241.

Rahner C, Mitic L, Anderson JM (2001). Heterogenity in expression and subcellu $\neg$ lar localization of Claudins 2, 3,4, and 5 in the rat liver, pancreas, and gut. Gastroenterology, 120, 411-22. 
Rangel LB, Agarwal R, D'Souza T, et al (2003). Tight junction proteins claudin-3 and claudin- 4 are frequently overexpressed in ovarian cancer but not in ovarian cystadenomas. Clin Cancer Res, 9, 2567-75.

Sheehan GM, Kallakury BV, Sheehan CE, et al (2007). Loss of claudins- 1 and -7 and expression of claudins-3 and -4 correlate with prognostic variables in prostatic adenocarcinomas. Hum Pathol, 38, 564-9.

Singh AB, Sharma A, Dhawan P(2010). Claudin family of proteins and cancer: an overview. J Oncol, 2010, 541957.

Soini Y, Takasawa A, Eskelinen M, et al (2012). Expression of claudins 7 and 18 in pancreatic ductal adenocarcinoma: association with features of differentiation. J Clin Pathol, $\mathbf{6 5}, 431-6$.

Szekely E, Torzsok P, Riesz P, et al (2011). Expression of claudins and their prognostic significance in noninvasive urothelial neoplasms of the human urinary bladder. $J$ Histochem Cytochem, 59, 932-41.

Tan C, Cruet-Hennequart S, Troussard A, et al (2004). Regulation of tumor angiogenesis by integrin-linked kinase (ILK). Cancer Cell, 5, 79-90.

Tsukahara M, Nagai H, Kamiakito T, et al (2005). Distinct expression patterns of claudin- 1 and claudin- 4 in intraductal papillary-mucinous tumors of the pancreas. Pathol Int, 55, 63-9.

Tsukita S, Furuse M, Itoh M (2001). Multifunctional strands in tight junctions. Nature Reviews Molecular Cell Biology, 2, 285-93.

Tsutsumi K, Sato N, Tanabe R, et al (2012). Claudin-4 expression predicts survival in pancreatic ductal adenocarcinoma. Ann Surg Oncol, 3, 491-9.

Usami Y, Chiba H, Nakayama F, et al (2006). Reduced expression of Claudin-7 cor-relates with invasion and metastasis in squamous cell carcinoma of the esophagus. Human Pathology, 37, 569-77.

Wang WJ, Qin SH, Zhang JW, et al (2014). Combination doxorubicin and interferon- $\alpha$ therapy stimulates immunogenicity of murine pancreatic cancer Panc02 cells via up-regulation of NKG2D ligands and MHC class I. Asian Pac J Cancer Prev, 15, 9667-72.

Westmoreland JJ, Drosos Y, Kelly J et al. (2012).Dynamic distribution of claudin proteins in pancreatic epithelia undergoing morphogenesis or neoplastic transformation. Dev Dyn, 241, 583-94.

Will C, Fromm M, Muller D (2008). Claudin tight junction proteins: novel aspects in paracellular transport. Perit Dial Int $, 28,577-84$.

Yeo TP, Hruban RH, Leach SD, et al (2002). Pancreatic cancer. Curr Probl Cancer, 26, 176-275.

Yoon CH, Kim MJ, Park MJ, et al (2010). Claudin-1 acts through c-Abl-protein kinase Cdelta (PKCdelta) signaling and has a causal role in the acquisition of invasive capacity in human liver cells. J Biol Chem, 285, 226-33. 